\title{
The impact of rheumatoid arthritis on the risk of adverse events following joint replacement: a real-world cohort study
}

This article was published in the following Dove Press journal:

Clinical Epidemiology

\author{
Edward Burn' \\ Christopher J Edwards ${ }^{2}$ \\ David W Murray' \\ Alan Silman' \\ Cyrus Cooper ${ }^{1,3}$ \\ Nigel K Arden ${ }^{1,3}$ \\ Daniel Prieto-Alhambra ${ }^{1,4}$ \\ Rafael Pinedo-Villanueva ${ }^{1,3}$ \\ 'Nuffield Department of \\ Orthopaedics, Rheumatology and \\ Musculoskeletal Sciences, University \\ of Oxford, Oxford, UK; ${ }^{2}$ NIHR \\ Wellcome Trust Clinical Research \\ Facility, University Hospital \\ Southampton, Southampton, UK; \\ ${ }^{3}$ MRC Lifecourse Epidemiology \\ Unit, Southampton University, \\ Southampton, UK; ${ }^{4}$ GREMPAL \\ Research Group, Idiap Jordi Gol and \\ CIBERFes, Universitat Autonoma \\ de Barcelona and Instituto de Salud \\ Carlos III, Barcelona, Spain
}

Purpose: To assess whether rheumatoid arthritis (RA) is associated with a greater risk of adverse events following total knee replacement (TKR) and total hip replacement (THR) than osteoarthritis (OA).

Patients and methods: Individuals with a diagnosis of RA or OA were identified using primary care records. TKR and THR following diagnosis were identified using linked hospital records. Myocardial infarction (MI), prosthetic joint infection (PJI), venous thromboembolism (VTE), and death were identified within 90 days following surgery, and revision procedures over 10 years following surgery. The impact of RA compared to OA on the risk for these adverse events was assessed using Cox proportional hazard models. Univariable models, with diagnosis as the only explanatory variable, and multivariable models, with age, gender, and year of surgery first added and then a measure of other comorbidities also included, were estimated.

Results: In all 20,763 individuals, with 10,260 TKR and 10,961 THR, were included in the analysis. Compared to those with OA, individuals with a diagnosis of RA had a greater incidence of MI over 90 days following TKR (OA: $0.28 \%$, RA: $0.75 \%$ ) and revision over 10 years following THR (OA: $5.55 \%$, RA: $8.68 \%$ ). Both of these differences were statistically significant with, for example, hazard ratios of 3.54 (1.44 to 8.73 ) for MI and 1.61 (1.06 to 2.46) for revision after controlling for age, gender, year of surgery, and other comorbidities.

Conclusion: These findings suggest that, compared to individuals with OA, those with RA have an increased short-term risk of MI following TKR. While risk of MI remains below 1\%, this does underline the importance of the management of cardiovascular risk factors for those with RA. RA was also associated with an increased long-term risk of revision following THR, which strengthens the argument for investing in therapies which may prevent the need for joint replacement.

Keywords: hip, knee, arthroplasty, osteoarthritis, surgery

\section{Introduction}

Total knee replacement (TKR) and total hip replacement (THR) typically result in substantial improvements in pain, function, and overall quality-of-life. ${ }^{1,2}$ As with any surgical procedure, however, there is a risk of adverse events. Potential perioperative complications for joint replacement include myocardial infarction (MI), venous thromboembolism (VTE), prosthetic joint infection (PJI), and even mortality. In addition, there is a long-term risk of revision following surgery, in which implant components are removed, added or exchanged.

Risks of adverse events following TKR and THR have mostly been estimated for individuals with osteoarthritis (OA), with OA being the most common indication for
Correspondence: Daniel Prieto-Alhambra Botnar Research Centre, NDORMS, University of Oxford, OX3 7HE, UK Email daniel.prietoalhambra@ndorms. ox.ac.uk 
surgery. A sizeable minority of patients undergoing TKR and THR, however, have inflammatory arthropathies, of which the most common is rheumatoid arthritis (RA). Outside of surgery, RA has been linked with increased risks for a range of health conditions. It is well-established that individuals with RA have an increased risk of cardiovascular disease (CVD), in particular ischemic heart disease, and related mortality compared to the general population. ${ }^{3-5}$ In addition, while the evidence is less conclusive, RA has also been linked with an increased general risk of $\mathrm{VTE}^{6}$ and a greater susceptibility for serious infections, due to both the disease itself and immunosuppressive treatments. ${ }^{7}$

While it is plausible that individuals with RA would also be at a higher risk of adverse events following joint replacement compared to those with OA, this relationship is not well understood. In this study, therefore, we assessed whether RA is associated with a greater risk of adverse events following TKR and THR compared to OA.

\section{Ethical approval}

Approval for the study was granted by the CPRD Independent Scientific Advisory Committee.

\section{Patients and methods Data}

Routinely-collected data from the English National Health Service (NHS) informed the analysis. Primary care practices within the Clinical Practice Research Datalink (CPRD) were linked to inpatient hospital records, provided by Hospital Episode Statistics Admitted Patient Care (HES APC), and mortality data, from the Office for National Statistics (ONS). HES APC data were extracted from 1997, the earliest year available, to 2014. CPRD data were extracted from 1995 to 2014 while ONS records were extracted from 1997 to 2014 to match HES APC.

\section{Study participants}

Individuals were identified separately for procedures relating to the knee and hip. For TKR, individuals with an incident (newly recorded) diagnosis of RA or knee OA were identified on the basis of clinical codes in CPRD. The first occurrence of a TKR following the index diagnosis was then identified using HES APC. A similar process was used for inclusion in the analysis of THR, with individuals required to have an incident diagnosis of hip OA or RA followed by the procedure.

Where an individual had a diagnosis of both RA and OA, it was not possible to determine which of their diagnoses drove the decision for joint replacement. As RA is a systemic inflammatory disease it was taken as the index diagnosis for the analyses.

Bilateral TKRs or THRs, where both the left and right joint were replaced simultaneously or staged, were excluded from the analysis due to the difficultly in establishing an appropriate time window and attributing adverse events as side of surgery was not consistently recorded. As the cohorts were established independently, the same individual could contribute both a TKR and a THR in the analysis.

In addition to identifying diagnoses, CPRD records also provided information on an individual's gender and year of birth. HES APC records contained information on individuals' primary procedure (TKR or THR), with the date of the procedure used to identify the year of surgery and, given an individual's year of birth recorded in CPRD, age at surgery. Diagnostic codes recorded for the primary procedure in HES APC were used to calculate an individual's Royal College of Surgeons (RCS) Charlson comorbidity score. ${ }^{8}$

\section{Adverse events}

Adverse events over 90 days (VTE, PJI, MI, and death) and 10 years (revision) following TKR and THR were identified using HES APC records. VTE and MI were identified on the basis of the primary diagnoses codes from the hospital episode (a period of hospital care under one consultant) in which the joint replacement occurred or any subsequent episode within the specified follow-up. PJI was identified by the corresponding ICD code as the primary diagnosis of hospital episodes and a concurrent or subsequent operation code either debridement, antibiotics, irrigation, and retention of the prosthesis or revision within a year of the diagnosis. ${ }^{9}$ Mortality records were based on data from the ONS.

Only incident adverse events were included in the analysis. If an individual had an event of interest recorded prior to their TKR or THR, they were excluded from the analysis of that event.

\section{Statistical methods}

The characteristics of individuals with an index diagnosis of OA or RA were compared in terms of standardized mean difference (SMD). An SMD of $<0.1$ indicates that there is minimal difference in the mean of a covariate between the two groups. ${ }^{10}$

The number of adverse events over 90 days (VTE, PJI, MI or death) and 10 years (revision) following surgery and the corresponding cumulative incidence, which gives the probability of experiencing the event within the time period under 
consideration before the occurrence of the competing risk of death (if mortality was not the adverse event in question), ${ }^{11}$ were estimated. These were compared for individuals with an index diagnosis of OA and those with a diagnosis of RA.

Cox proportional hazards regression models were then used to assess the association between RA and observed adverse events over 90 days (VTE, PJI, MI or death) and 10 years (revision) following surgery, relative to OA. Aside from mortality itself, other adverse events had mortality as a competing risk. Cause-specific hazard ratios (HRs) were therefore estimated, along with 95\% CIs, for non-fatal events and overall HRs for mortality. For each outcome, follow-up ended at the earliest of: a second TKR or THR, the end of data linkage with CPRD or date of data extraction, death, or transfer out. Models were only estimated if there were more than five events in the RA group.

Univariable models were first estimated with only diagnosis of RA or OA included. Subsequently, age, gender, and year of surgery were also included as explanatory factors. Finally, to control for the effect of other comorbidities on the risk of adverse events, the RCS Charlson score was also included (with codes for RA excluded to avoid double counting its effect). Further, the RCS Charlson score was dichotomized as 0 and $1+$ due to relatively small numbers of patients with multiple comorbidities. Proportionality was considered through a visual assessment of Schoenfeld residuals.

Data analysis was undertaken in $\mathrm{R} 3.3 .1,{ }^{12}$ with dplyr ${ }^{13}$ used for data manipulation, survival ${ }^{14}$ for fitting regression models and ggplot $2^{15}$ for producing cumulative incidence plots.

\section{Sensitivity analyses}

A sensitivity analysis was undertaken where adverse events were considered over the year following joint replacement, rather than within only the first 90 days. The analysis was also re-run excluding individuals who had both a diagnosis of RA and OA recorded.

\section{Results Study participants}

In all 20,763 individuals informed the analysis with 458 contributing to both the TKR and THR analyses. In total, 10,260 TKR (9,409 knee OA and 851 RA) and 10,961 THR (10,322 hip OA and 639 RA) were included. A flow chart summarizing inclusion into the study is provided in the Supplementary materials. Individuals' characteristics are detailed in Table 1. The number and cumulative incidence of adverse events following TKR and THR are detailed by diagnosis in Table
2. As there were fewer than five events for those with RA, regression models were not estimated for PJI following TKR and THR and MI following THR. The cumulative incidences of revision over 10 years following TKR and THR are shown in Figure 1.

\section{Adverse events following TKR}

There was a higher incidence of MI over the 90 days following TKR among individuals with RA compared to those with OA (RA: $0.75 \%$, OA: $0.28 \%$ ), (Table 2). This difference was significant in regression models, such as after adjustment for age, gender, and year of surgery (HR: 3.57 [95\% CI: 1.45 to 8.80]), (Table 3).

Year of surgery being closer to the end of follow-up was associated with a significantly reduced risk of death (HR: 0.90 [0.84 to 0.97$]$ ). Higher age at surgery and male gender were also associated with significantly increased risk of 90-day MI (HRs: 1.11 [1.06 to 1.16] and 3.88 [1.76 to 8.55], respectively, from full multivariable regressions). Higher age at surgery and comorbidity (RCS Charlson score of $1+$ ) were associated with an increased risk of 90-day postoperative mortality (HRs: 1.09 [1.05 to 1.14] and 2.65 [1.45 to 4.82] from full multivariable regressions). Older age at surgery was associated with a reduced risk of revision over the 10 years following TKR (HR: 0.96 [0.94 to 0.97]), while male gender was associated with an increased risk (HR: 1.32 [1.04 to 1.68], from full multivariable regressions). Fully specified models are provided in the Supplementary materials.

\section{Adverse events following THR}

There was a higher incidence of revision over the 10 years following THR among individuals with RA compared to those with OA (RA: 8.68\%, OA: 5.55\%), (Table 2). This difference was significant in regression models, such as after adjustment for age, gender, and year of surgery (HR: 1.64 [95\% CI: 1.08 to 2.50]), (Table 3).

Year of surgery being closer to the end of follow-up was associated with a significantly reduced risk of VTE and death (HRs: 0.91 [0.87 to 0.95 ] and 0.89 [0.84 to 0.94], respectively). Higher age at surgery, male gender, and comorbidity (RCS Charlson score of $1+$ ) were each associated with a significantly increased risk of 90-day mortality following THR (HRs: 1.12 [1.09 to 1.15], 1.81 [1.12 to 2.94], and 2.52 [1.53 to 4.15] from full multivariable regressions, respectively). Older age at surgery was associated with a reduced risk of revision over the 10 years following THR (HR: 0.99 [0.98 to 1.00 ] from the fully specified regression). 
Table I Characteristics of study participants

\begin{tabular}{|c|c|c|c|c|c|c|}
\hline & \multicolumn{3}{|l|}{ THR } & \multicolumn{3}{|l|}{ TKR } \\
\hline & OA & RA & SMD & OA & RA & SMD \\
\hline $\mathrm{n}$ & 9,409 & 851 & & 10,322 & 639 & \\
\hline Age (median [IQR]) & $71.0[64.0,77.0]$ & $69.0[61.5,75.0]$ & 0.21 & $70.0[62.0,77.0]$ & $70.0[62.0,76.0]$ & 0.06 \\
\hline Gender: female (n [\%]) & $5240[55.7]$ & $594[69.8]$ & 0.29 & $5957[57.7]$ & $479[75.0]$ & 0.37 \\
\hline Year of surgery (median [IQR]) & $2009[2006,2011]$ & $2008[2005,2011]$ & 0.19 & $2008[2005,2011]$ & $2008[2005,2011]$ & 0.03 \\
\hline \multicolumn{7}{|l|}{ Diagnoses recorded in HES APC } \\
\hline Myocardial infarction (n [\%]) & $127[1.3]$ & $\mathrm{II}[1.3]$ & 0.01 & $|3|[1.3]$ & $9[1.4]$ & 0.01 \\
\hline Congestive cardiac failure (n [\%]) & $88[0.9]$ & $8[0.9]$ & $<0.01$ & $127[1.2]$ & $10[1.6]$ & 0.03 \\
\hline Peripheral vascular disease ( $\mathrm{n}[\%])$ & $93[1.0]$ & $8[0.9]$ & $<0.01$ & $95[0.9]$ & $7[1.1]$ & 0.02 \\
\hline Cerebrovascular disease (n [\%]) & $52[0.6]$ & $2[0.2]$ & 0.05 & $52[0.5]$ & $4[0.6]$ & 0.02 \\
\hline Dementia (n [\%]) & $21[0.2]$ & $4[0.5]$ & 0.04 & $32[0.3]$ & $5[0.8]$ & 0.06 \\
\hline Chronic pulmonary disease (n [\%]) & $986[10.5]$ & $105[12.3]$ & 0.06 & 918 [8.9] & $80[12.5]$ & 0.12 \\
\hline Rheumatological disease* (n [\%]) & $75[0.8]$ & $24[2.8]$ & 0.15 & $105[1.0]$ & $19[3.0]$ & 0.14 \\
\hline Liver disease (n [\%]) & $19[0.2]$ & $\mathrm{I}[0.1]$ & 0.02 & $20[0.2]$ & $2[0.3]$ & 0.02 \\
\hline Diabetes mellitus ( $\mathrm{n}[\%]$ ) & $933[9.9]$ & $58[6.8]$ & 0.11 & 708 [6.9] & $47[7.4]$ & 0.02 \\
\hline Hemiplegia or paraplegia (n [\%]) & $18[0.2]$ & $0[0.0]$ & 0.06 & $15[0.1]$ & $2[0.3]$ & 0.04 \\
\hline Renal disease (n [\%]) & $183[1.9]$ & $19[2.2]$ & 0.02 & $222[2.2]$ & $13[2.0]$ & 0.01 \\
\hline Any malignancy (n [\%]) & $74[0.8]$ & $4[0.5]$ & 0.04 & $92[0.9]$ & $5[0.8]$ & 0.01 \\
\hline Metastatic solid tumor (n [\%]) & $6[0.1]$ & $0[0.0]$ & 0.04 & $8[0.1]$ & $2[0.3]$ & 0.05 \\
\hline AIDS HIV infection (n [\%]) & 0 & 0 & 0 & I $[0.0]$ & $0[0.0]$ & 0.01 \\
\hline RCS Charlson* (n [\%]) & & & 0.06 & & & 0.14 \\
\hline 0 & $7161[76.1]$ & $643[75.6]$ & & $8203[79.5]$ & $474[74.2]$ & \\
\hline I & $1876[19.9]$ & $174[20.4]$ & & $1777[17.2]$ & $|3|[20.5]$ & \\
\hline 2 & $320[3.4]$ & $32[3.8]$ & & $282[2.7]$ & $29[4.5]$ & \\
\hline $3+$ & $52[0.6]$ & $2[0.2]$ & & $60[0.6]$ & $5[0.8]$ & \\
\hline
\end{tabular}

Note: *Codes for RA were omitted from the calculation of the rheumatological disease category and for the overall RCS Charlson score.

Abbreviations: TKR, total knee replacement; THR, total hip replacement; OA, osteoarthritis; RA, rheumatoid arthritis; SMD, standardized mean difference; IQR, interquartile range; n, number; HES APC, Hospital Episode Statistics Admitted Patient Care; RCS, Royal College of Surgeons.

Table 2 Adverse events following TKR and THR

\begin{tabular}{|c|c|c|c|c|}
\hline & \multicolumn{2}{|l|}{ OA } & \multicolumn{2}{|l|}{ RA } \\
\hline & Number of events & Cumulative incidence (\%) & Number of events & Cumulative incidence (\%) \\
\hline \multicolumn{5}{|l|}{ TKR } \\
\hline \multicolumn{5}{|l|}{ 90-day } \\
\hline VTE & 79 & $0.86(0.67$ to 1.05$)$ & 5 & $0.6 \mathrm{I}(0.07$ to $\mathrm{I} . \mathrm{I} 4)$ \\
\hline PJI & 17 & 0.19 (0.10 to 0.27$)$ & 2 & 0.23 (0.00 to 0.56$)$ \\
\hline MI & 24 & 0.28 (0.17 to 0.39$)$ & 6 & $0.75(0.15$ to 1.34$)$ \\
\hline Death & 43 & $0.46(0.33$ to 0.60$)$ & 3 & $0.36(0.00$ to 0.76$)$ \\
\hline \multicolumn{5}{|l|}{ 10-year } \\
\hline Revision & 248 & 5.73 (4.63 to 6.82$)$ & 18 & 5.07 ( 1.04 to 8.94$)$ \\
\hline \multicolumn{5}{|l|}{ THR } \\
\hline \multicolumn{5}{|l|}{ 90-day } \\
\hline VTE & 106 & I.05 (0.85 to I.25) & 5 & 0.79 (0.10 to 1.48$)$ \\
\hline PJI & 12 & $0.12(0.05$ to 0.18$)$ & 2 & $0.32(0.00$ to 0.76$)$ \\
\hline MI & 31 & $0.32(0.21$ to 0.44$)$ & 1 & 0.16 (0.00 to 0.48$)$ \\
\hline Death & 61 & $0.60(0.45$ to 0.75$)$ & 7 & I.II (0.29 to I.92) \\
\hline \multicolumn{5}{|l|}{ 10-year } \\
\hline Revision & 255 & 5.55 (4.68 to $6.4 I)$ & 24 & 8.68 (4.27 to 12.88$)$ \\
\hline
\end{tabular}

Note: Number and cumulative incidence of VTE, PJI, MI, and death following TKR and THR split by index diagnosis of OA or RA.

Abbreviations: TKR, total knee replacement; THR, total hip replacement; OA, osteoarthritis; RA, rheumatoid arthritis; VTE, venous thromboembolism; PJI, prosthetic joint infection; MI, myocardial infarction.

\section{Sensitivity analysis}

Assessing adverse events over 1 year rather than 90 days led to broadly similar findings (details provided in $\underline{\text { Supplementary }}$ materials). As in the primary analysis, a diagnosis of RA was associated with a significantly increased risk of MI following TKR (HR: 3.02 [1.68 to 5.41] from the model adjusted for age, 


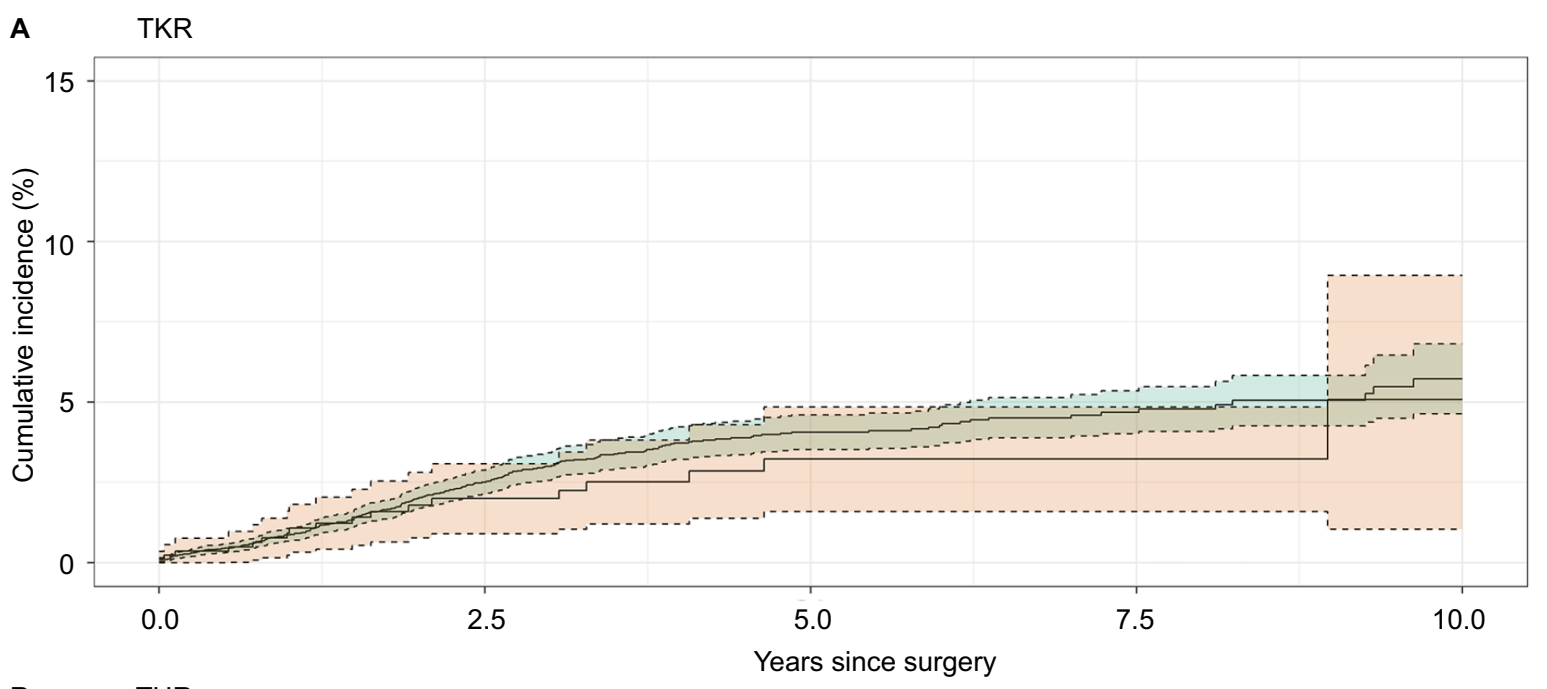

B THR

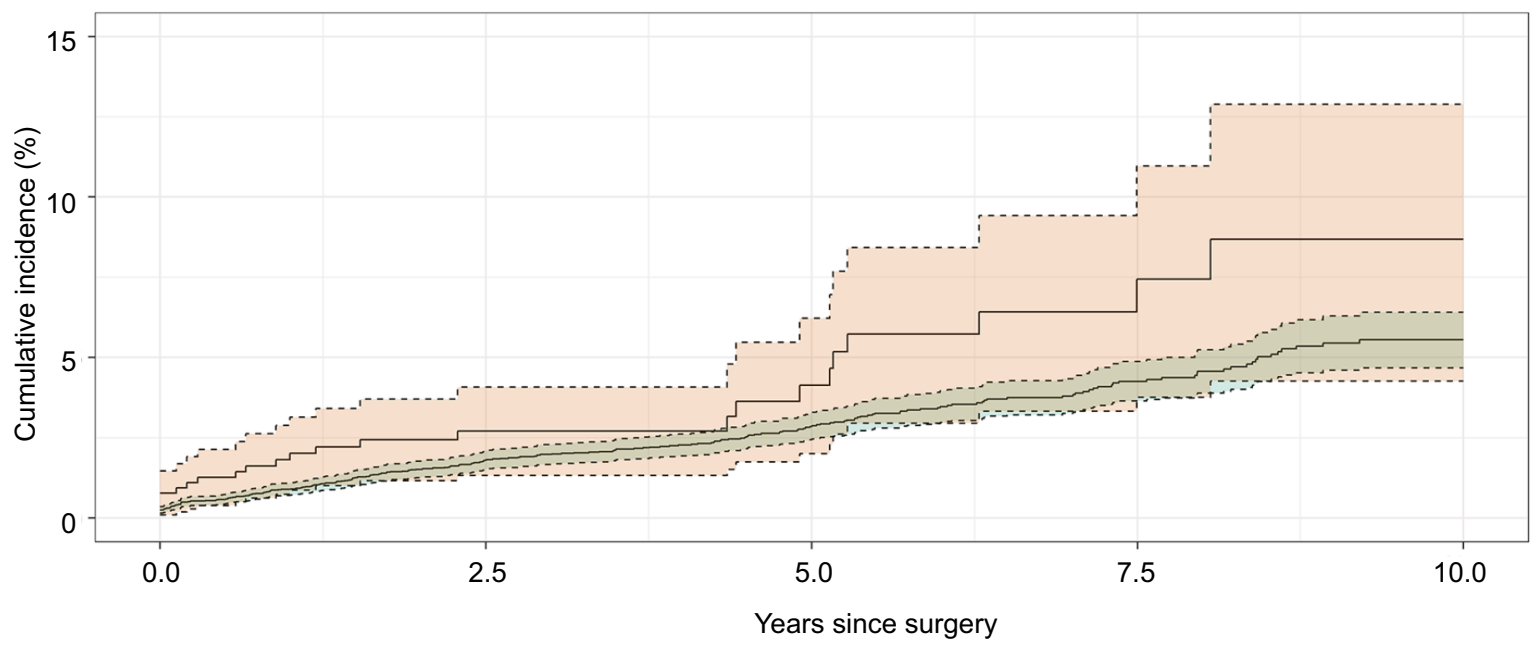

Figure I Cumulative incidence of revision.

Notes: Observed cumulative incidence of revision following (A) total knee replacement (TKR) and (B) total hip replacement (THR), stratified by diagnosis of rheumatoid arthritis (RA) or osteoarthritis (OA).

gender, and year of surgery). In addition, RA was also associated with significantly increased risk of death after THR over the year following surgery (HR: 2.60 [1.62 to 4.17] from the model adjusted for age, gender, and year of surgery). The results were broadly similar after excluding individuals with both a diagnosis of RA and OA, with RA still associated with an increased risk of revision (HR: 1.72 [1.07 to 2.78] from the unadjusted model). However, while the cumulative incidence of MI following TKR was still higher for RA, there were too few events to run the regression model to assess the effect of other explanatory factors (see Supplementary materials for further details).

\section{Discussion}

\section{Key results}

Individuals with a diagnosis of RA had a greater incidence of MI over 90 days following TKR compared to those with OA
(OA: $0.28 \%$, RA: $0.75 \%$ ), with this difference being statistically significant after controlling for age, gender, and year of surgery (HR: 3.57 [1.45 to 8.80]). Patients with RA were also more likely to undergo a revision surgery over the 10 years following THR than individuals with OA (OA: 5.55\%, RA: $8.68 \%$ ), with this difference also statistically significant after controlling for age, gender, and year of surgery (HR: 1.64 [1.08 to 2.50$])$.

\section{Study findings in context}

In our study, a prior diagnosis of RA was not associated with significant differences in the risk for VTE over 90 days or 1 year following TKR or THR. Previous studies have also found patients with RA not to be at a significantly increased risk of VTE following joint replacement compared to those with OA. ${ }^{16,17}$ While estimates were not significant in this study, 
Table 3 Association between RA and risk of post-operative adverse events

\begin{tabular}{|c|c|c|c|}
\hline & \multicolumn{3}{|c|}{ Estimated effect of RA compared to OA, hazard ratio $(95 \% \mathrm{Cl})$} \\
\hline & Unadjusted model & $\begin{array}{l}\text { Multivariable model with age, } \\
\text { gender, and year of surgery } \\
\text { included }\end{array}$ & $\begin{array}{l}\text { Multivariable model with age, } \\
\text { gender, year of surgery, and RCS } \\
\text { Charlson* included }\end{array}$ \\
\hline \multicolumn{4}{|l|}{ TKR } \\
\hline \multicolumn{4}{|l|}{ 90-day } \\
\hline VTE & $0.70(0.29$ to 1.74$)$ & $0.7 \mathrm{I}(0.29$ to $\mathrm{I} .76)$ & $0.70(0.28$ to 1.74$)$ \\
\hline PJI & - & - & - \\
\hline MI & 2.69 ( 1.10 to 6.59$)$ & $3.57(1.45$ to 8.80$)$ & 3.54 ( 1.44 to 8.73$)$ \\
\hline Death & 0.77 (0.24 to 2.47$)$ & 0.85 (0.26 to 2.74$)$ & $0.82(0.25$ to 2.64$)$ \\
\hline \multicolumn{4}{|l|}{ 10-year } \\
\hline Revision & $0.79(0.49$ to 1.28$)$ & 0.71 (0.43 to I.I4) & $0.7 \mathrm{I}(0.44$ to $\mathrm{I} .15)$ \\
\hline \multicolumn{4}{|l|}{ THR } \\
\hline \multicolumn{4}{|l|}{ 90-day } \\
\hline VTE & $0.77(0.31$ to 1.89$)$ & $0.74(0.30$ to $I .83)$ & $0.74(0.30$ to $\mathrm{I} .8 \mathrm{I})$ \\
\hline PJI & - & - & - \\
\hline MI & - & - & - \\
\hline Death & $1.85(0.85$ to 4.05$)$ & 2.31 (1.05 to 5.07$)$ & 2.17 (0.98 to 4.77$)$ \\
\hline \multicolumn{4}{|l|}{ 10-year } \\
\hline Revision & 1.63 (1.07 to 2.48$)$ & I.64 (I.08 to 2.50$)$ & I.6I (I.06 to 2.46$)$ \\
\hline
\end{tabular}

Notes: Hazard ratios associated with diagnosis of RA relative to OA for VTE, MI, and death following TKR and THR. There were too few PJIs following TKR and THR and MI following THR for meaningful regression analysis to be undertaken. Full model specifications are reported in the Supplementary materials. *Codes for RA were omitted from the calculation of the RCS Charlson score and the score was dichotomized as 0 and $\mathrm{I}+$.

Abbreviations: TKR, total knee replacement; THR, total hip replacement; OA, osteoarthritis; RA, rheumatoid arthritis; VTE, venous thromboembolism; PJI, prosthetic joint infection; MI, myocardial infarction; RCS, Royal College of Surgeons.

younger age at surgery has previously been associated with a significantly lower risk of VTE following THR. ${ }^{17}$

In this study, there were insufficient cases of PJI to assess whether there was a significant difference between those with $\mathrm{OA}$ and RA. However, previous research has found RA to be associated with an increased risk of PJI compared to OA. Over the year following surgery, for example, RA was found to be associated with sub-hazard ratio (SHR) of 1.46 (1.131.88).$^{18}$ This increased risk could be caused by a reduced immune system response due to RA or by the immunosuppressive treatments being taken. ${ }^{19}$ Younger age at surgery and additional comorbidities have previously been associated with a significantly lower risk of infection following THR. ${ }^{17}$

A prior diagnosis of RA was associated with a significantly increased 90-day risk of MI following TKR compared to OA in our data. There were too few cases of MI, however, to estimate whether there was a difference following THR. Despite the high cardiovascular risk profile of RA patients, and the known peak in risk of MI following joint replacement, ${ }^{20}$ few studies have assessed whether those with RA are at higher risk of cardiovascular events following joint replacement compared to those with OA. One study, however, previously found RA to be associated with an increased risk of cardiovascular death within 6 weeks of joint replacement compared to those without a diagnosis of OA, (odds ratio [OR] of $1.50[0.96-2.33]) .{ }^{21}$ In line with the findings from this study, older age and comorbidities have also previously been found to be associated with significantly higher risks of cardiac events following both TKR and THR. ${ }^{22}$

In this analysis, we found no significant increase in risk of mortality over 90 days following either TKR or THR for those with RA. RA, however, was associated with significantly higher mortality over 1 year following THR. RA has been found to be associated with an increased risk of mortality over 90 days following THR (OR: 1.88 [1.17 to 3.03]), ${ }^{17}$ over 6 weeks (OR: 1.85 [1.09 to 3.13]), ${ }^{21}$ and 1 year (HR: 1.25 [1.01 to 1.55$])^{18}$ following joint replacement in general. A diagnosis of RA is also associated with increased risk of mortality compared to OA in general. ${ }^{23}$ Consequently, the increased risk observed following surgery for RA is not necessarily attributable to the receipt of surgery but may reflect underlying differences in baseline risk of mortality. Consistent with the findings from this study, older age, comorbidities, and male gender have been found to be associated with an increased risk of 90- and 45-day mortality following THR and TKR, respectively. ${ }^{24,25}$

We found RA, compared to OA, to be associated with a significantly increased 10 -year risk of revision following THR. RA has previously been associated with an increased risk of hip dislocation following THR. ${ }^{26,27}$ Hip dislocation, one of the most common reasons for early revision following THR, may explain the increased risk of revision estimated 
in this study. Findings from previous studies, however, are mixed. Studies have found patients with RA not to be at a significantly increased long-term risk of revision following THR, ${ }^{26,28}$ a reduced risk of revision for reasons other than infection (SHR: 0.71 [0.57 to 0.89]), ${ }^{18}$ and also an increased risk of revision for infection following TKR (relative risk: 1.6 [1.06 to 2.38]). ${ }^{29}$ The effect of RA therefore seems to vary depending on the reason for revision. Moreover, the mixed findings regarding long-term risks of revision have likely been influenced by study design and, in particular, whether the competing risk of mortality was accounted for. Aside from a recent study, ${ }^{18}$ the competing risk of mortality has not been taken into account in most previous analyses. In line with previous studies, younger age at surgery was associated with an increased risk of revision following both TKR and THR. ${ }^{30}$ Male gender was associated with an increased risk of revision following TKR in this study and has previously been associated with an increased risk following both TKR and THR in the UK. ${ }^{31}$

\section{Strengths and limitations of this study}

This study was informed by routinely collected data. The primary concern with such data is typically the accuracy of coding, given that data collection is not undertaken primarily for research purposes. Where possible, therefore, validated codes were used in this study. However, while the codes used to identify diagnoses of RA in CPRD have previously been validated, ${ }^{32}$ the $\mathrm{OA}$ codes have not. It can be expected that although the specificity of OA diagnosis will have been high, given that individuals went on to have joint replacement, the sensitivity may have been less so. Consequently, there is a concern as to how representative those with OA in this study are of the population in general.

Despite the potential limitations, the key strength of this study was the use of linked primary care and hospital data. This allowed for the identification of diagnoses using primary care data and procedures and subsequent adverse events from hospital records. In addition, the use of routinely collected data has allowed for the inclusion of a large "real-world" study population, sufficient to test for important differences in risks of rare adverse events.

\section{Study implications}

Individuals with RA are at increased risk of MI over 90 days following knee replacement compared to those with OA. As with the general increased risk of cardiovascular events for those with RA, this is likely due to both the nature of the disease itself and medications used in its treatment. While in absolute terms the observed incidence of $\mathrm{MI}$ remained $<1 \%$, this finding underlines the importance of the management of cardiovascular risk factors for those with RA, for example through the use of anti-TNF and methotrexate treatments, particularly prior to TKR. ${ }^{4}$

Compared to OA, RA has also been found in this study to be associated with an increased risk of revision over the 10 years following THR. With a cumulative incidence of over $8 \%$ for those with RA, compared to $5 \%$ for those with $\mathrm{OA}$, the additional revisions represent a significant cost to providers and burden of ill-health for individuals. This will only strengthen the argument for investing in available therapies which may delay or entirely prevent the need for joint replacement for those with RA.

\section{Data access and data sharing}

CPRD data with HES linkage were provided under a license that does not permit sharing. Data are obtainable from CPRD subject to a full application.

\section{Acknowledgments}

The authors would like to thank Miss Susan Thwaite (National Rheumatoid Arthritis Society) for her role as the patient and public representative and her role in the study steering committee.

DPA is funded by a National Institute for Health Research Clinician Scientist award (CS-2013-13-012). This article presents independent research funded by the National Institute for Health Research (NIHR). The views expressed are those of the authors and not necessarily those of the NHS, the NIHR, or the Department of Health. This work was supported by the NIHR Biomedical Research Centre, Oxford.

\section{Author contributions}

EB, CJE, DWM, AS, CC, NKA, DPA, and RPV all made substantial contributions to conception and design of the study. EB, RPV, and DPA undertook the statistical analysis. EB, RPV, and DPA drafted the manuscript with CJE, DWM, AS, $\mathrm{CC}$, and NKA revising it for important intellectual content. All authors gave final approval of the version to be published, and agree to be accountable for all aspects of the work.

\section{Disclosure}

NKA has received personal fees from Freshfields Bruckhaus Deringer, Bioventus, Flexion, Merck, and Regeneron, all outside the submitted work. DPA reports grants from Amgen, Servier, and UCB Biopharma, and non-financial support from Amgen, all outside the submitted work. DWM reports 
grants and personal fees from Zimmer Biomet. In addition, DWM has various patents related to Unicompartmental Knee Replacement (Zimmer Biomet) with royalties paid, all outside the submitted work. The authors report no other conflicts of interest in this work.

\section{References}

1. Pivec R, Johnson AJ, Mears SC, Mont MA. Hip arthroplasty. Lancet. 2012;380(9855):1768-1777.

2. Carr AJ, Robertsson O, Graves S, et al. Knee replacement. Lancet. 2012;379(9823):1331-1340.

3. Crowson CS, Liao KP, Davis JM 3rd, et al. Rheumatoid arthritis and cardiovascular disease. Am Heart J. 2013;166(4):622-628.e1.

4. Peters MJ, Symmons DP, McCarey D, et al. EULAR evidence-based recommendations for cardiovascular risk management in patients with rheumatoid arthritis and other forms of inflammatory arthritis. Ann Rheum Dis. 2010;69(2):325-331.

5. Avina-Zubieta JA, Choi HK, Sadatsafavi M, et al. Risk of cardiovascular mortality in patients with rheumatoid arthritis: a meta-analysis of observational studies. Arthritis Rheum. 2008;59(12):1690-1697.

6. Bacani AK, Gabriel SE, Crowson CS, Heit JA, Matteson EL. Noncardiac vascular disease in rheumatoid arthritis: increase in venous thromboembolic events? Arthritis Rheum. 2012;64(1):53-61.

7. Listing J, Gerhold K, Zink A. The risk of infections associated with rheumatoid arthritis, with its comorbidity and treatment. Rheumatology (Oxford). 2013;52(1):53-61.

8. Armitage JN, van der Meulen JH; Royal College of Surgeons Comorbidity Consensus Group. Identifying co-morbidity in surgical patients using administrative data with the Royal College of Surgeons Charlson Score. Br J Surg. 2010;97(5):772-781.

9. Graves N, Wloch C, Wilson J, et al. A cost-effectiveness modelling study of strategies to reduce risk of infection following primary hip replacement based on a systematic review. Health Technol Assess. 2016;20(54):1-144.

10. Austin PC. An introduction to propensity score methods for reducing the effects of confounding in observational studies. Multivariate Behav Res. 2011;46(3):399-424.

11. Austin PC, Lee DS, Fine JP. Introduction to the analysis of survival data in the presence of competing risks. Circulation. 2016;133(6):601-609.

12. R Core Team. R: A Language and Environment for Statistical Computing. Vienna, Austria: R Foundation for Statistical Computing; 2016.

13. Wickham H, Francois R. dplyr: A Grammar of Data Manipulation. R package version 0.5.0. 2016.

14. Therneau T. A Package for Survival Analysis in S. 2015.

15. Wickham H. ggplot2: Elegant Graphics for Data Analysis. New York: Springer-Verlag; 2009.

16. Niki Y, Matsumoto H, Hakozaki A, Mochizuki T, Momohara S. Rheumatoid arthritis: a risk factor for deep venous thrombosis after total knee arthroplasty? Comparative study with osteoarthritis. J Orthop Sci. 2010;15(1):57-63.

17. SooHoo NF, Farng E, Lieberman JR, Chambers L, Zingmond DS. Factors that predict short-term complication rates after total hip arthroplasty. Clin Orthop Relat Res. 2010;468(9):2363-2371.

Clinical Epidemiology

\section{Publish your work in this journal}

Clinical Epidemiology is an international, peer-reviewed, open access, online journal focusing on disease and drug epidemiology, identification of risk factors and screening procedures to develop optimal preventative initiatives and programs. Specific topics include: diagnosis, prognosis, treatment, screening, prevention, risk factor modification, Submit your manuscript here: https://www.dovepress.com/clinical-epidemiology-journal
18. Cordtz RL, Zobbe K, Højgaard P, et al. Predictors of revision, prosthetic joint infection and mortality following total hip or total knee arthroplasty in patients with rheumatoid arthritis: a nationwide cohort study using Danish healthcare registers. Ann Rheum Dis. 2018;77(2):281-288.

19. Stundner O, Danninger T, Chiu YL, et al. Rheumatoid arthritis vs osteoarthritis in patients receiving total knee arthroplasty: perioperative outcomes. J Arthroplasty. 2014;29(2):308-313.

20. Lalmohamed A, Vestergaard P, Klop C, et al. Timing of acute myocardial infarction in patients undergoing total hip or knee replacement: a nationwide cohort study. Arch Intern Med. 2012;172(16):1229-1235.

21. Tropea J, Brand CA, Bohensky M, Van Doornum S. Myocardial infarction and mortality following joint surgery in patients with rheumatoid arthritis: a retrospective cohort study. Arthritis Res Ther. 2016;18:69.

22. Singh JA, Jensen MR, Harmsen WS, Gabriel SE, Lewallen DG. Cardiac and thromboembolic complications and mortality in patients undergoing total hip and total knee arthroplasty. Ann Rheum Dis. 2011;70(12):2082-2088.

23. Watson DJ, Rhodes T, Guess HA. All-cause mortality and vascular events among patients with rheumatoid arthritis, osteoarthritis, or no arthritis in the UK General Practice Research Database. J Rheumatol. 2003;30(6):1196-1202.

24. Hunt LP, Ben-Shlomo Y, Clark EM, et al. 90-day mortality after 409 096 total hip replacements for osteoarthritis, from the National Joint Registry for England and Wales: a retrospective analysis. Lancet. 2013;382(9898):1097-1104.

25. Hunt LP, Ben-Shlomo Y, Clark EM, et al. 45-day mortality after 467779 knee replacements for osteoarthritis from the National Joint Registry for England and Wales: an observational study. Lancet. 2014;384(9952):1429-1436.

26. Ravi B, Escott B, Shah PS, et al. A systematic review and metaanalysis comparing complications following total joint arthroplasty for rheumatoid arthritis versus for osteoarthritis. Arthritis Rheum. 2012;64(12):3839-3849.

27. Conroy JL, Whitehouse SL, Graves SE, et al. Risk factors for revision for early dislocation in total hip arthroplasty. $J$ Arthroplasty. 2008;23(6):867-872.

28. Rud-Sørensen C, Pedersen AB, Johnsen SP, Riis AH, Overgaard S. Survival of primary total hip arthroplasty in rheumatoid arthritis patients. Acta Orthop. 2010;81(1):60-65.

29. Schrama JC, Espehaug B, Hallan G, et al. Risk of revision for infection in primary total hip and knee arthroplasty in patients with rheumatoid arthritis compared with osteoarthritis: a prospective, population-based study on 108,786 hip and knee joint arthroplasties from the Norwegian Arthroplast. Arthritis Care Res (Hoboken). 2010;62(4):473-479.

30. Wainwright C, Theis JC, Garneti N, Melloh M. Age at hip or knee joint replacement surgery predicts likelihood of revision surgery. J Bone Joint Surg Br. 2011;93(10):1411-1415.

31. Culliford D, Maskell J, Judge A, Arden NK; COAST Study group. A population-based survival analysis describing the association of body mass index on time to revision for total hip and knee replacements: results from the UK general practice research database. BMJ Open. 2013;3(11):e003614.

32. Thomas SL, Edwards CJ, Smeeth L, Cooper C, Hall AJ. How accurate are diagnoses for rheumatoid arthritis and juvenile idiopathic arthritis in the general practice research database? Arthritis Rheum. 2008;59(9):1314-1321.

systematic reviews, risk and safety of medical interventions, epidemiology and biostatistical methods, and evaluation of guidelines, translational medicine, health policies and economic evaluations. The manuscript management system is completely online and includes a very quick and fair peer-review system, which is all easy to use. 